OPEN ACCESS

Edited by:

Tao Huang,

Peking University, China

Reviewed by:

Hualiang Lin,

Sun Yat-sen University, China

Zilong Zhang,

Sun Yat-sen University, China

*Correspondence:

Peige Song

peigesong@zju.edu.cn

Yimin Zhu

zhuym@zju.edu.cn

${ }^{t}$ These authors have contributed equally to this work

Specialty section:

This article was submitted to

Systems Endocrinology,

a section of the journal

Frontiers in Endocrinology

Received: 03 September 2021

Accepted: 28 October 2021

Published: 26 November 2021

Citation:

Yu J, Yi Q, Hou L, Chen G, Shen Y, Song $Y$, Zhu $Y$ and Song $P$ (2021) Transition of Lipid Accumulation Product Status and the Risk of Type 2 Diabetes Mellitus in Middle-Aged and Older Chinese: A National Cohort Study.

Front. Endocrinol. 12:770200. doi: 10.3389/fendo.2021.770200

\section{Transition of Lipid Accumulation Product Status and the Risk of Type 2 Diabetes Mellitus in Middle-Aged and Older Chinese: A National Cohort Study}

\author{
Jinyue $\mathrm{Yu}^{1,2 \dagger}$, Qian $\mathrm{Yi}^{1+}$, Leying Hou ${ }^{1}$, Ge Chen ${ }^{3}$, Yaojia Shen ${ }^{1}$, Yuan Song ${ }^{4}$, \\ Yimin $\mathrm{Zhu}^{5^{*}}$ and Peige Song ${ }^{1 *}$ \\ ${ }^{1}$ School of Public Health and Women's Hospital, Zhejiang University School of Medicine, Hangzhou, China, ${ }^{2}$ Institute of \\ Child Health, University College London, London, United Kingdom, ${ }^{3}$ Medical Research and Biometrics Center, National \\ Center for Cardiovascular Diseases, Beijing, China, ${ }^{4}$ School of Nursing, Henan University of Traditional Chinese Medicine, \\ Zhengzhou, China, ${ }^{5}$ Department of Epidemiology and Biostatistics, School of Public Health, Zhejiang University School of \\ Medicine, Hangzhou, China
}

Background: Lipid accumulation product (LAP), a product of waist circumference (WC) and fasting triglycerides (TG), is a measure of lipid accumulation and an effective predictor of metabolic syndrome. This study aimed to evaluate the associations of LAP and its longitudinal transitions with type 2 diabetes mellitus (T2DM) among middle-aged and older Chinese.

Methods: Data were extracted from the China Health and Retirement Longitudinal Study (2011, 2013, 2015, and 2018). LAP was defined as (WC-65) ×TG for men, and (WC-58) $\times T G$ for women. Participants were classified into high- and low-LAP groups at baseline, and subsequently into four transition patterns during 2011-2015: maintained-high, maintained-low, high-to-low, and low-to-high LAP. The longitudinal transition patterns of LAP on the development of T2DM were assessed by multivariable Cox frailty models.

Results: Overall, 7397 participants were included for analysis, among whom 849 (11.5\%) developed T2DM between 2011 and 2018. Women with high-LAP levels at baseline presented a higher risk of T2DM (hazard ratios $[\mathrm{HR}]=1.37,95 \%$ confidence interval $[\mathrm{Cl}]$ : 1.07-1.77), while no significant association was found in men. Compared with women with maintained-low LAP pattern, those with transition patterns of low-to-high LAP and maintained-high LAP were at higher risk of T2DM ( $H R=1.99$ and 1.98 , both $P<0.05)$; however, for men, the significantly positive association was only observed in maintainedhigh LAP transition pattern (HR=1.53, 95\% Cl: 1.04-2.23).

Conclusions: Elevated LAP levels and the transition patterns of maintained-high LAP and low-to-high LAP are significant risk factors for T2DM in women. Preventions are needed to combat T2DM at an early dyslipidemic stage.

Keywords: lipid accumulation product (LAP), type 2 diabetes mellitus, risk factors, transition, China 


\section{INTRODUCTION}

Diabetes mellitus (DM) is a disease of abnormal glucose metabolism that affects multiple organ systems. Globally, DM has become a high-risk factor for morbidity and mortality (1). According to the International Diabetes Federation, the number of people affected by DM globally in 2019 was 463 million, among whom an estimated 232 million people had undiagnosed DM (2). Type 2 diabetes mellitus (T2DM) accounts for over $90 \%$ of patients with DM, bringing profound psychological and physical distress to patients and putting a huge burden on health care systems (3). Evidence shows that the incidence and prevalence of T2DM vary by geographical region, with $79 \%$ of patients living in low-to-middle-income countries (2). The overall prevalence of T2DM in China increased sharply from $1.3 \%$ in $1980-1989$ to $4.5 \%$ in $1990-1999,6.8 \%$ in $2000-2009$, and $8.7 \%$ in 2010-2014. Previous research estimated that by 2025 , the prevalence of T2DM in China would increase to $12.5 \%$ (4), becoming a serious epidemic that produces considerable socioeconomic pressures on both individuals and the society.

A 2017 Lancet review shows that $60 \%$ of patients with T2DM are obese (body-mass index $[\mathrm{BMI}] \geq 30 \mathrm{~kg} / \mathrm{m}^{2}$ ) (3). In China, a population-based survey with 5071 subjects aged 40 years or older in Shanghai demonstrated that obese people had a significantly higher risk of T2DM (5). Apart from obesity, individuals with normal weight but increased visceral adipose tissue content are also characterized by the presence of insulin resistance and impaired glucose tolerance, which are strongly associated with the development of T2DM $(4,6)$. In the current era when the association between high BMI and the morbidity of T2DM has been well demonstrated, research interests may need to focus more on the measurement of visceral adiposity, especially when visceral adiposity may represent a higher risk of T2DM (7).

Traditional techniques, such as magnetic resonance imaging (MRI) and computed tomography (CT), are currently the gold standard for measuring visceral adiposity (8). However, those are not suitable for routine clinical practice due to high costs, intensive labor, and the hazard posed by the use of radiation. Therefore, an alternative continuous index of visceral adiposity, the lipid accumulation product (LAP), has been proposed. Based on a combination of waist circumference (WC) and fasting triglycerides (TG), LAP could accurately reflect visceral adiposity and be easily obtained (9). A bulk of studies found that compared with conventional obesity indices, such as BMI, waist-to-hip ratio (WHR) and waist-to-height ratio (WHtR), LAP presented a better predictive ability in metabolic syndrome, $\mathrm{DM}$, and impaired fasting glucose (10-12).

Previous studies have reported the efficacy of LAP in identifying visceral adiposity and insulin resistance $(8,13)$. However, there is a lack of studies investigating the association of LAP and the risk of T2DM among middle-aged and older Chinese (5). Moreover, whether the dynamic transition of LAP across years is associated with the development of T2DM is still poorly understood. Therefore, this study aimed to evaluate the effect of LAP and its transition on the development of T2DM in middle to older aged adults based on a national Chinese cohort study.

\section{MATERIAL AND METHODS}

\section{Data and Sample}

Data were extracted from the China Health and Retirement Longitudinal Study (CHARLS, available at http://charls.pku.edu. $\mathrm{cn} / \mathrm{en}$ ). CHARLS is a national survey in middle to older aged adults in China (aged 45 years and above) that attempts to provide a wealth of information ranging from socio-economic status to health conditions. The survey informs scientific research and priority setting related to the middle-aged and older adult population in China $(14,15)$.

The national baseline survey of CHARLS was fielded in 20112012. A total of 10257 households and 17708 individuals from 150 counties/districts and 450 villages/urban communities were involved across the country. The geographic regions of investigated provinces are presented in Figure $\mathbf{S 1}$ and Table S1. The sampling strategy utilized for the CHARLS Survey involved multiple steps. First, all counties/districts were stratified according to region, urban/rural setting and economic conditions, and 150 counties/districts were randomly selected. Then, three primary sampling units (PSU) were randomly selected from each county, namely rural administrative villages and urban communities. Finally, the "CHARLS-GIS" software was used to map at least 24 families within each PSU, in which residents aged 45 years or older were interviewed with his/her spouse through a face-to-face personal interview in June 2011 and were followed up every two years. To date, CHARLS has been conducted for five rounds from 2011 to 2018. Blood tests were conducted in 2011 and 2015.

In total, 17708 participants were successfully interviewed in four rounds of the CHARLS Survey (2011, 2013, 2015 and 2018), with a family response rate of $80.5 \%$. Individuals who were aged $\geq 45$ years, with complete blood sample and biomarker data, and non-diabetic at baseline were included $(\mathrm{n}=9485)$. After excluding individuals with no follow-up response $(\mathrm{n}=$ $531)$, age $<45$ years $(\mathrm{n}=194)$, and incomplete data of LAP at baseline ( $n=1363)$, a total of 7397 subjects were included in the final analysis (Figure S2). The demographic, socio-economic, geographical and behavioral characteristics of the included and excluded subjects are outlined in Table S2.

This study was approved by the ethics review committee of Peking University and carried out by the National School for Development (China Centre for Economic Research) of Peking University. All participants signed written informed consent.

\section{Measurements}

From 2011 to 2018, trained interviewers collected information on demographics, geographic location, socio-economic status, health-related behaviors, and medical history through structured questionnaires. Anthropometry data were gathered following a standard protocol from the World Health Organization (16). Body weight was measured to the nearest $0.1 \mathrm{~kg}$ (in light clothes and without shoes) using a digital scale (Omron, HN-286). Height was measured to the nearest $0.1 \mathrm{~cm}$ without shoes on a stadiometer (Seca Corporation, 213). WC was horizontally measured at the middle point of the line between the lower rib and the upper iliac crest without wearing a coat. Blood pressure (BP) was measured at the right arm of the participants using an 
electronic sphygmomanometer (Omron, hem-7200) at intervals of 45 seconds. Systolic (SBP) and diastolic blood pressures (DBP) were measured three times in the seated position after 10 minutes of rest by use of a sphygmomanometer.

Venous blood samples were collected by professional nurses after fasting for at least 12 hours at night. The whole blood count was performed immediately at the survey sites. The whole blood samples were then stored at 4 degrees Celsius, and the remaining samples were transported to the central Laboratory in Beijing Youanmen Centre for Clinical Laboratory of Capital Medical University for further laboratory analysis $(14,15)$. The levels of blood glucose, total cholesterol (TC), TG, low density lipoprotein (LDL-C) and high density lipoprotein (HDL-C) were tested by enzyme colorimetry. The Glycosylated hemoglobin (HbA1c) levels were measured by boronated-affinity high-performance liquid chromatography.

\section{Definitions of Covariates}

The tertiles of the natural logarithm of per capita expenditures (ln $[\mathrm{PCE}]$ ) were used as indicators of family wealth, with the bottom, middle and top tertiles representing poor, middle, and rich status, respectively $(17,18)$. Participants' residence was classified into North China, Northeast China, East China, South-central China, Southwest China and Northwest China (see Figure S1 and Table S1 for more details). Hypertension was defined by an $\mathrm{SBP} \geq 140 \mathrm{mmHg}$, and/or a $\mathrm{DBP} \geq 90 \mathrm{mmHg}$, and/or a self-reported physician diagnosis, and/or currently with antihypertensive drugs, and/or under other related therapeutic measures $(18,19)$. Participants were categorized as normal, overweight or obese based on BMI, with cut-off points at 24 $\mathrm{kg} / \mathrm{m}^{2}$ and $28 \mathrm{~kg} / \mathrm{m}^{2}(20)$.

\section{Definitions of LAP and T2DM}

LAP was defined as $(\mathrm{WC}(\mathrm{cm})-65) \times($ TG concentration $(\mathrm{mmol} / \mathrm{l}))$ for men, and $(\mathrm{WC}(\mathrm{cm})-58) \times(\mathrm{TG}$ concentration $(\mathrm{mmol} / \mathrm{l}))$ for women (9). This formula included the minimum WC values (65 and $58 \mathrm{~cm}$ for men and women, respectively) used to define a gender-specific starting point in the Third National Health and Nutrition Examination (NHANES III) (9). In the present study, Participants were classified into high- and low-LAP groups at baseline using cut-off points obtained with the baseline receiver operating characteristics analysis, and then subsequently into four transition patterns during follow-up (2011-2015): maintainedhigh, maintained-low, high-to-low, and low-to-high LAP. The cut-off points of LAP for the diagnosis of T2DM were 18.86 (51.3\% specificity and $67.7 \%$ sensitivity) for men and $40.53(70.2 \%$ specificity and $51.1 \%$ sensitivity) for women (Figure S3).

The diagnosis of T2DM was established using criteria from the American Diabetes Association: fasting blood glucose $\geq 126$ $\mathrm{mg} / \mathrm{dL}$ ( $7.0 \mathrm{mmol} / \mathrm{L})$, and/or random blood glucose $\geq 200 \mathrm{mg} / \mathrm{dL}$ (11.1 mmol/L), and/or $\mathrm{HbA} 1 \mathrm{c} \geq 6.5 \%$, and/or self-reported diagnosis, and/or currently under hypoglycemic therapy (21).

\section{Statistical Analysis}

Statistical analysis was conducted using SAS statistical software (version 9.4; SAS Institute Inc., Cary, NC, USA). Differences in continuous and categorical variables across groups were assessed by Mann-Whitney test and Chi-square test. Person-years were calculated from the date of baseline interview and physical examination (CHARLS 2011) until the occurrence of T2DM events or death or the time when he/she was censored or the end of follow-up (CHARLS 2018), whichever came first. The cumulative incidence rates of T2DM were calculated by the Kaplan-Meier method. Cox frailty models with random effect and adjustments, were used to explore associations between LAP level and new onset T2DM by gender and setting (rural and urban). Model 1 adjusted for age. Model 2 adjusted for education, marital status, ln (PCE), region, hypertension, smoking, drinking, and general obesity based on Model 1. Model 3 adjusted for TC, LDL-C and HDL-C based on Model 2. In addition, the effects of four transition patterns of LAP on T2DM were assessed by multivariable Cox frailty models. A multiple imputation method was used to impute missing data of baseline characteristics. A total of 945 subjects were imputed for missing data (Table S3).

\section{RESULTS}

\section{Demographic Characteristics}

The baseline characteristics of the included participants stratified by gender, setting (rural and urban) and LAP transition pattern are shown in Table 1 and Table S4. In total, 7397 participants (men=3447, women $=3950$ ) were involved in cohort analysis. No significant gender differences were observed between rural and urban areas. More women than men were illiterate in both rural and urban settings; the proportion of people with a low education level in rural areas was significantly higher than that in urban settings. The baseline LAP score was 25.46 (interquartile range [IQR]: 14.39-44.41). Totally, 4202 (56.8\%) individuals were with low-LAP (men=1734, women=2468) while 3195 (43.2\%) were identified as high LAP at baseline (men=1713, women=1482). Significant differences in LAP status were observed between genders in both rural and urban settings. The proportion of general obesity and hypertension was significantly higher in women than in men. Higher plasma HDL and TG were more observed in women compared to men in both rural and urban settings $(P<0.05)$.

\section{Association Between Baseline LAP and T2DM}

Figure 1 shows the cumulative incidence of T2DM for low- and high-baseline LAP status stratified by setting and gender, from 2011 to 2018. Overall, the cumulative incidence of T2DM was highest in women with high-LAP at baseline (17.95\%). Association between baseline LAP status and the risk of newonset T2DM is shown in Table 2 and Table S5. Positive associations between incident T2DM and high-LAP status at baseline were observed in both genders, with a crude hazard ratios (HR) of 1.78 (95\% confidence interval [CI]: 1.43-2.22) for men and $=2.00(95 \% \mathrm{CI}: 1.68-2.39)$ for women. After full adjustments, the association was still significant in women ( $\mathrm{HR}=1.37,95 \% \mathrm{CI}: 1.07-1.77)$ but insignificant in men (HR = 1.29, 
TABLE 1 | Demographic, socioeconomic and geographic characteristics of the included participants at baseline (CHARLS 2011).

\begin{tabular}{|c|c|c|c|c|c|c|c|}
\hline \multirow[t]{2}{*}{ Characteristics } & \multirow[t]{2}{*}{ Overall $(n=7,397)$} & \multicolumn{2}{|c|}{ Male } & \multirow[t]{2}{*}{$P^{a}$} & \multicolumn{2}{|c|}{ Female } & \multirow[t]{2}{*}{$\mathbf{P}^{\mathrm{b}}$} \\
\hline & & $\begin{array}{c}\text { Rural } \\
\text { (n=2,353) }\end{array}$ & $\begin{array}{c}\text { Urban } \\
(n=1,094)\end{array}$ & & $\begin{array}{c}\text { Rural } \\
\text { (n=2,626) }\end{array}$ & $\begin{array}{c}\text { Urban } \\
(n=1,324)\end{array}$ & \\
\hline Age group & & & & 0.131 & & & 0.156 \\
\hline 45-49 years & $1437(19.4 \%)$ & $369(15.7 \%)$ & $182(16.6 \%)$ & & $566(21.5 \%)$ & $320(24.2 \%)$ & \\
\hline 50-59 years & $2641(35.7 \%)$ & 819(34.8\%) & 416(38.1\%) & & $942(35.9 \%)$ & $464(35.0 \%)$ & \\
\hline $60-69$ years & 2182(29.5\%) & $748(31.8 \%)$ & $326(29.8 \%)$ & & $759(28.9 \%)$ & $349(26.4 \%)$ & \\
\hline$\geq 70$ years & $1137(15.4 \%)$ & $417(17.7 \%)$ & $170(15.5 \%)$ & & $359(13.7 \%)$ & 191(14.4\%) & \\
\hline Education & & & & $<0.001$ & & & $<0.001$ \\
\hline Illiterate & 2173(29.4\%) & $393(16.7 \%)$ & $74(6.7 \%)$ & & $1318(50.2 \%)$ & $388(29.3 \%)$ & \\
\hline Literate & 1403(19.0\%) & 496(21.1\%) & 203(18.6\%) & & 488(18.6\%) & 216(16.3\%) & \\
\hline Primary education & $1661(22.5 \%)$ & $699(29.7 \%)$ & 259(23.7\%) & & $450(17.1 \%)$ & 253(19.1\%) & \\
\hline Middle school & 2159(29.1\%) & $764(32.5 \%)$ & $558(51.0 \%)$ & & $370(14.1 \%)$ & $467(35.3 \%)$ & \\
\hline Marital status & & & & $<0.001$ & & & 0.035 \\
\hline Married or cohabiting & 6518(88.1\%) & 2108(89.6\%) & 1035(94.6\%) & & 2266(86.3\%) & 1109(83.8\%) & \\
\hline Single & $879(11.9 \%)$ & $245(10.4 \%)$ & $59(5.4 \%)$ & & $360(13.7 \%)$ & $215(16.2 \%)$ & \\
\hline $\operatorname{Ln}(P C E)$ & & & & $<0.001$ & & & $<0.001$ \\
\hline Bottom tertile & 2164(33.3\%) & $801(37.9 \%)$ & 223(23.3\%) & & $887(38.4 \%)$ & 253(22.6\%) & \\
\hline Middle tertile & 2166(33.3\%) & 704(33.3\%) & 326(34.0\%) & & $749(32.5 \%)$ & $387(34.6 \%)$ & \\
\hline Top tertile & 2166(33.3\%) & $607(28.7 \%)$ & 409(42.7\%) & & $672(29.1 \%)$ & $478(42.8 \%)$ & \\
\hline Region of China & & & & $<0.001$ & & & 0.001 \\
\hline North & $950(12.9 \%)$ & $342(14.5 \%)$ & $120(11.0 \%)$ & & $328(12.4 \%)$ & $160(12.0 \%)$ & \\
\hline Northeast & $503(6.8 \%)$ & $136(5.8 \%)$ & $92(8.4 \%)$ & & 167(6.4\%) & 108(8.2\%) & \\
\hline East & $2221(30.0 \%)$ & 678(28.8\%) & $345(31.5 \%)$ & & 793(30.2\%) & 405(30.6\%) & \\
\hline South Central & 1747(23.6\%) & 486(20.7\%) & 294(26.9\%) & & $614(23.4 \%)$ & $353(26.7 \%)$ & \\
\hline Southwest & 1366(18.5\%) & $475(20.2 \%)$ & 194(17.7\%) & & 474(18.1\%) & 223(16.8\%) & \\
\hline Northwest & 610(8.2\%) & 236(10.0\%) & $49(4.5 \%)$ & & 250(9.5\%) & $75(5.7 \%)$ & \\
\hline General Obesity $^{c}$ & & & & $<0.001$ & & & $<0.001$ \\
\hline Normal & $3657(50.5 \%)$ & $1452(62.9 \%)$ & 497(46.2\%) & & 1226(47.8\%) & 482(37.3\%) & \\
\hline Overweight & $2748(37.9 \%)$ & $715(30.9 \%)$ & $461(42.8 \%)$ & & 1007(39.2\%) & $565(43.8 \%)$ & \\
\hline Obese & $840(11.6 \%)$ & $144(6.2 \%)$ & 118(11.0\%) & & $334(13.0 \%)$ & 244(18.9\%) & \\
\hline Hypertension ${ }^{c}$ & & & & 0.009 & & & 0.011 \\
\hline Normal & 4394(59.5\%) & 1473(62.8\%) & 634(58.1\%) & & 1559(59.5\%) & $728(55.2 \%)$ & \\
\hline Hypertension & $2986(40.5 \%)$ & 874(37.2\%) & 458(41.9\%) & & $1063(40.5 \%)$ & $591(44.8 \%)$ & \\
\hline Smoking & & & & $<0.001$ & & & 0.030 \\
\hline No Smoking & 4506(61.1\%) & $538(23.0 \%)$ & $323(29.7 \%)$ & & 2441(93.1\%) & 1204(91.1\%) & \\
\hline Smoking & 2869(38.9\%) & 1805(77.0\%) & 766(70.3\%) & & $181(6.9 \%)$ & 117(8.9\%) & \\
\hline Drinking $^{c}$ & & & & 0.859 & & & 0.007 \\
\hline No Drinking & 5089(68.8\%) & $1046(44.5 \%)$ & 483(44.1\%) & & 2345(89.3\%) & 1215(92.0\%) & \\
\hline Drinking & 2304(31.2\%) & $1306(55.5 \%)$ & $611(55.9 \%)$ & & 281(10.7\%) & 106(8.0\%) & \\
\hline TC & & & & 0.291 & & & 0.489 \\
\hline$\leq 200 \mathrm{mg} / \mathrm{dL}$ & $4577(61.9 \%)$ & $1602(68.1 \%)$ & $725(66.3 \%)$ & & $1506(57.3 \%)$ & $744(56.2 \%)$ & \\
\hline$>200 \mathrm{mg} / \mathrm{dL}$ & 2820(38.1\%) & 751(31.9\%) & 369(33.7\%) & & $1120(42.7 \%)$ & $580(43.8 \%)$ & \\
\hline HDL & & & & $<0.001$ & & & $<0.001$ \\
\hline$\geq 50 \mathrm{mg} / \mathrm{dL}$ & $3730(50.4 \%)$ & $1179(50.1 \%)$ & 437(39.9\%) & & $1460(55.6 \%)$ & $654(49.4 \%)$ & \\
\hline$<50 \mathrm{mg} / \mathrm{dL}$ & $3667(49.6 \%)$ & $1174(49.9 \%)$ & $657(60.1 \%)$ & & $1166(44.4 \%)$ & $670(50.6 \%)$ & \\
\hline LDL & & & & 0.001 & & & 0.253 \\
\hline$\leq 100 \mathrm{mg} / \mathrm{dL}$ & $3214(43.5 \%)$ & $1199(51.0 \%)$ & $489(44.7 \%)$ & & 1031(39.3\%) & 495(37.4\%) & \\
\hline$>100 \mathrm{mg} / \mathrm{dL}$ & 4183(56.5\%) & $1154(49.0 \%)$ & $605(55.3 \%)$ & & 1595(60.7\%) & $829(62.6 \%)$ & \\
\hline TG & & & & $<0.001$ & & & 0.012 \\
\hline$\leq 150 \mathrm{mg} / \mathrm{dL}$ & $5667(76.6 \%)$ & 1925(81.8\%) & 824(75.3\%) & & 1973(75.1\%) & $945(71.4 \%)$ & \\
\hline$>150 \mathrm{mg} / \mathrm{dL}$ & $1730(23.4 \%)$ & $428(18.2 \%)$ & $270(24.7 \%)$ & & $653(24.9 \%)$ & $379(28.6 \%)$ & \\
\hline LAP SCORE & & & & $<0.001$ & & & $<0.001$ \\
\hline Median (IQR) & 25.46(14.39-44.41) & 16.90(9.67-30.91) & 23.56(12.89-41.87) & & $30.10(18.47-49.10)$ & $35.59(20.91-57.75)$ & \\
\hline LAP & & & & $<0.001$ & & & $<0.001$ \\
\hline Low LAP & $4202(56.8 \%)$ & $1303(55.4 \%)$ & 431(39.4\%) & & $1719(65.5 \%)$ & $749(56.6 \%)$ & \\
\hline High LAP & 3195(43.2\%) & $1050(44.6 \%)$ & $663(60.6 \%)$ & & $907(34.5 \%)$ & $575(43.4 \%)$ & \\
\hline
\end{tabular}

Data were presented as $n$ (\%) or median with interquartile range (IQR); ${ }^{a}$ Comparison between rural males and urban males; ${ }^{b}$ comparison between rural females and urban females; ${ }^{c}$ data for some participants were missing; PCE, per capita expenditures; LAP, lipid accumulation product.

95\% CI: 0.96-1.74). When further stratified by setting, fully adjusted models revealed significant associations between baseline highLAP and T2DM only in rural women, with HR being $1.60(95 \%$ CI: 1.18-2.17).

\section{Association Between LAP Transition and T2DM}

In terms of LAP transitions, it was found that the cumulative incidence of T2DM was the highest in participants with 
A

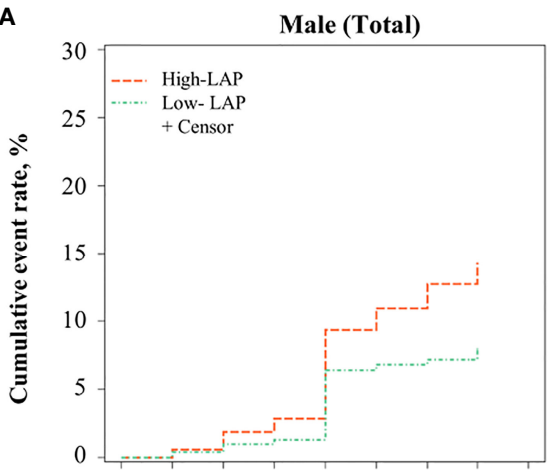

20112012201320142015201620172018 Number of cumulative events $\begin{array}{lllllllll}\text { High-LAP } & 0 & 10 & 32 & 48 & 154 & 177 & 203 & 225\end{array}$ $\begin{array}{llllllllll}\text { Low-LAP } & 0 & 7 & 17 & 22 & 106 & 112 & 117 & 128\end{array}$

C

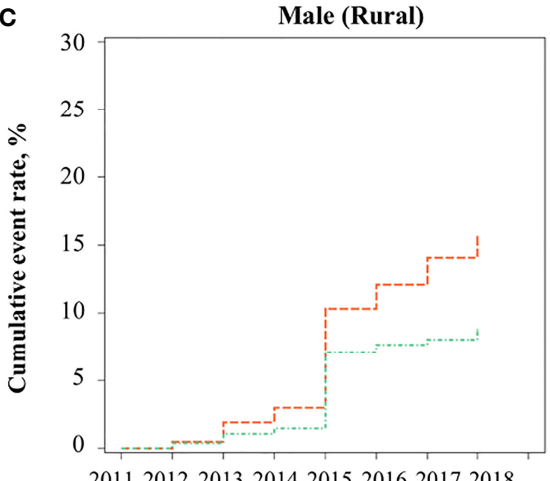

20112012201320142015201620172018 Number of cumulative events $\begin{array}{lllllllll}\text { High-LAP } & 0 & 5 & 20 & 31 & 105 & 121 & 139 & 153\end{array}$ $\begin{array}{lllllllll}\text { Low-LAP } & 0 & 5 & 14 & 19 & 89 & 95 & 99 & 107\end{array}$

E

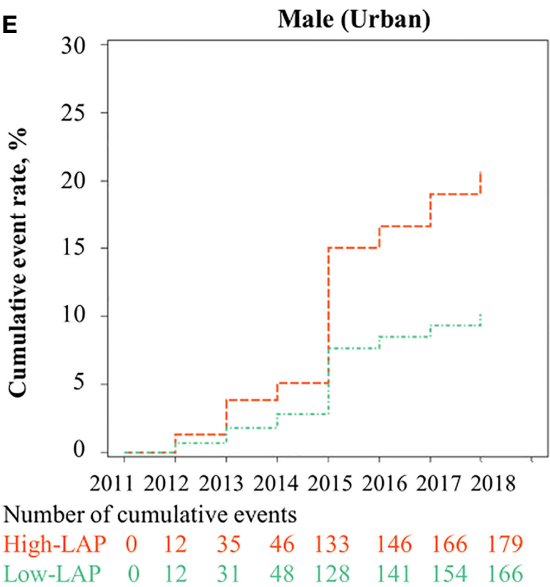

B

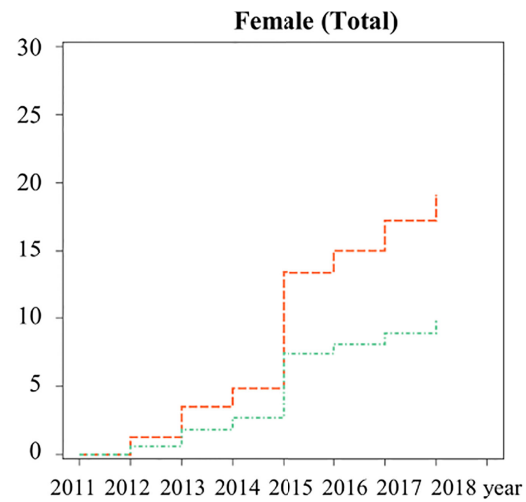

Number of cumulative events

$\begin{array}{lllllllll}\text { High-LAP } & 0 & 19 & 52 & 71 & 192 & 213 & 242 & 266\end{array}$

$\begin{array}{lllllllll}\text { Low-LAP } & 0 & 15 & 45 & 66 & 178 & 193 & 211 & 230\end{array}$

D

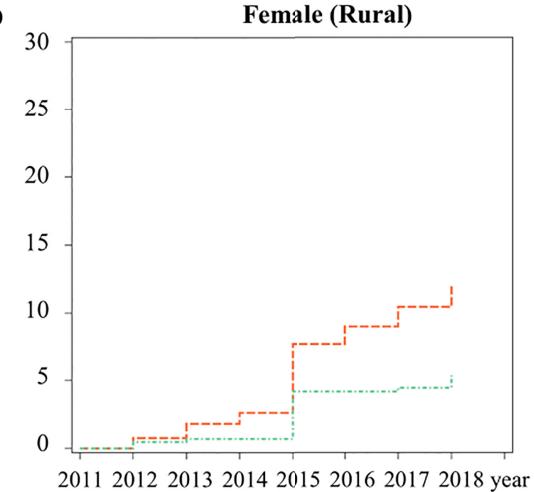

Number of cumulative events

$\begin{array}{lllllllll}\text { High-LAP } & 0 & 5 & 12 & 17 & 49 & 56 & 64 & 72\end{array}$

$\begin{array}{lllllllll}\text { Low-LAP } & 0 & 2 & 3 & 3 & 17 & 17 & 18 & 21\end{array}$

$\mathbf{F}$

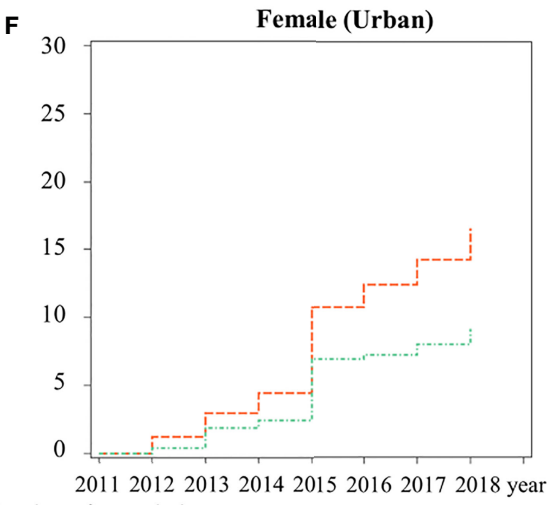

Number of cumulative events

$\begin{array}{lllllllll}\text { High-LAP } & 0 & 7 & 17 & 25 & 59 & 67 & 76 & 87\end{array}$

$\begin{array}{lllllllll}\text { Low-LAP } & 0 & 3 & 14 & 18 & 50 & 52 & 57 & 64\end{array}$

FIGURE 1 | Cumulative incidence of T2DM for LAP phenotypes (Low- and High-) stratified by urban/rural settings and sex from CHARLS 2011 to 2018. LAP, lipid accumulation product; The cumulative event rate of T2DM was significantly different between low-LAP and high-LAP in (A) Male, (B) Female, (C) Male in rural, (D) Female in rural, (E) Male in urban, and (F) Female in urban.

maintained-high (Figure 2). Figure 3 reports the risk of T2DM by LAP transition pattern stratified by gender after adjusting for age, education, region, hypertension, smoking, drinking, general obesity, TC, LDL-C and HDL-C. Overall, compared to individual whose LAP level was maintained low from baseline to follow-up, a 1.5 to 2.0 -fold risk of T2DM was observed in people with maintained-high LAP pattern $\left(\mathrm{HR}_{\mathrm{men}}=1.53,95 \mathrm{CI} \%: 1.04-2.23\right.$, $\mathrm{HR}_{\text {women }}=1.98,95 \%$ CI: 1.43-2.75, $\left.P<0.05\right)$, and a two-fold risk 
TABLE 2 | Hazard ratios for T2DM by LAP phenotypes in middle-aged and older Chinese, CHARLS 2011-2018.

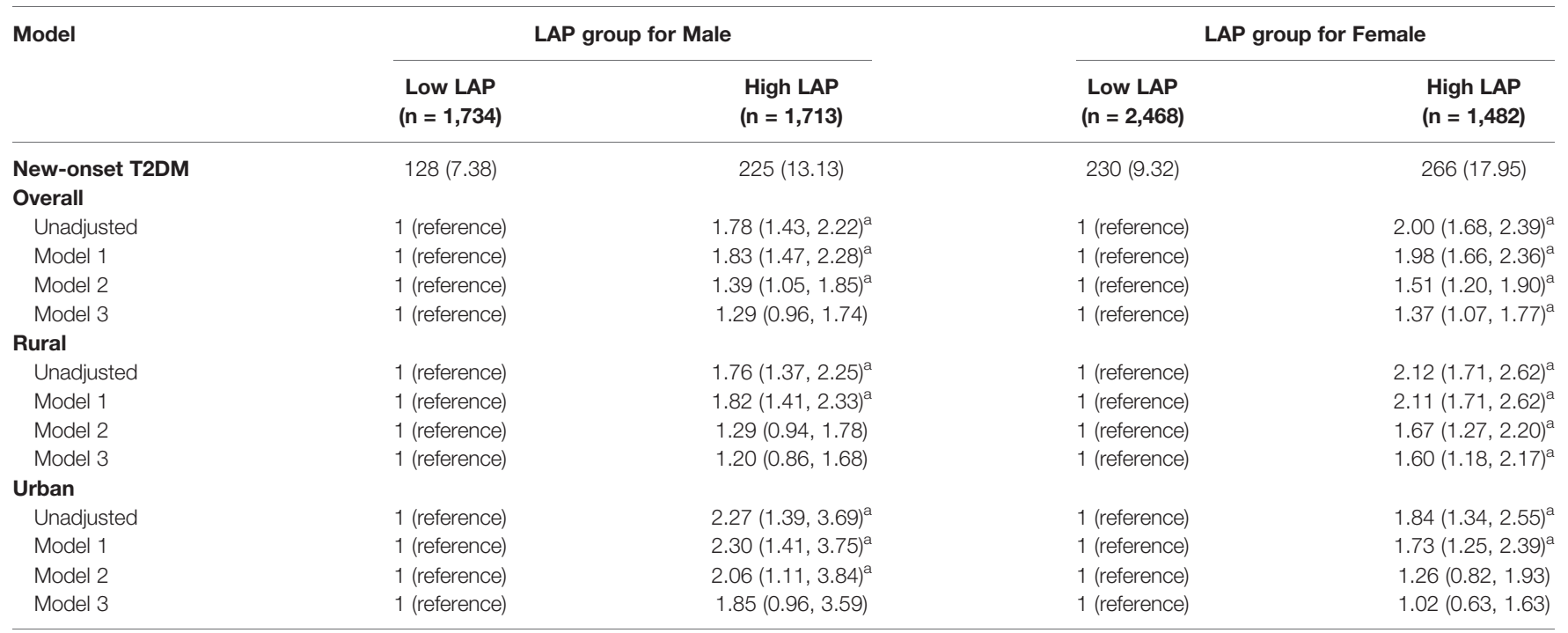

${ }^{a} P<0.05$.Data were presented as $n(\%)$ or hazard ratios (95\% Cl); Associations between LAP and the risk of T2DM were assessed using multivariable Cox frailty models with random intercepts to account for clustering of participants by city; T2DM, type 2 diabetes mellitus; LAP, lipid accumulation product.

Model 1 was adjusted for age. Model 2 was adjusted for education, marital status, Ln(PCE), region, hypertension, smoking, drinking, and general obesity based on Model 1. Model 3 was adjusted for TC, LDL-C and HDL-C based on Model 2.

of T2DM was observed in women whose LAP level was transferred from low to high $(\mathrm{HR}=1.99,95 \% \mathrm{CI}: 1.46-2.71)$. Comparatively, male participants with three transition patterns (low-to-high, high-to-low, and maintained-high) showed no significant difference in the risk of T2DM in both urban and rural settings, compared to those who have maintained-low LAP pattern during the follow-up. Similar results were found in Tables S6, S7 for data with multiple imputations.

\section{DISCUSSION}

In this prospective cohort study, we confirmed that higher LAP level was a significant risk factor for T2DM, especially in women. Women with high-LAP status at baseline presented a higher risk of T2DM. When assessing the relationship between LAP transitions and T2DM, women with maintained-high LAP pattern and low-to-high transition pattern were at an almost two-fold risk of T2DM compared to maintained-low LAP group. However, no such association was generally found in men, except for the maintained-high LAP pattern.

A number of studies have reported the rapid increase in the prevalence of DM in the Chinese adult population (22-24). The disease burden of DM in China is likely the result of population aging, urbanization, unhealthy diets, reduced exercise, and the consequent epidemic of obesity (25). Although visceral fat has been found to be independently associated with insulin resistance and could be used to estimate the risk of T2DM, the measurement for body visceral adiposity tissue volumes is not easy to conduct. MRI and CT have been considered the gold standard for visceral adiposity measurements, but both are not suitable for large epidemiological studies due to high costs and inconvenience. Comparatively, LAP can be easily measured in large-scale epidemiological studies and has been suggested as a useful surrogate marker of visceral adiposity. Previous studies have demonstrated that LAP has a similar or greater capacity to predict T2DM when compared with common fatness indices, such as BMI, WC, WHR and WHtR (10-12). For example, a six-year cohort study showed that LAP performed as good as BMI, WHR, and WHtR when predicting T2DM (26). Findings from this study were consistent with previous results, which indicated the positive effects of LAP on the prediction of DM in both genders $(9,26-28)$. Furthermore, in a recent cohort study examining the potential of LAP as an indicator for the development of T2DM among 15717 Chinese individuals (28), results showed a significantly increased risk of T2DM development in high LAP groups.

As for the effects of LAP by gender and by rural/urban setting, this study observed that the cumulative incidence of T2DM in women with high-LAP status was the highest. Similar results were found in a large population-based study in Japan (29), which reported the odds ratio for DM in subjects with high LAP was higher in women $(\mathrm{OR}=19.09)$ than in men $(\mathrm{OR}=7.40)$. A recent meta-analysis demonstrated a higher prevalence of T2DM in rural Chinese women than that in men (30). The difference in T2DM prevalence between genders was presumably explained by two reasons. The first might be the difference in sex steroid hormones and glucose homeostasis (31). Since the fat mass in healthy women is higher than that in men, the circulating free fatty acids and intramuscular fat content would consequently be higher (32), which could therefore induce insulin resistance in women. Another explanation might be the lack of physical activity in women compared to men. A regional study in China showed that rural men presented a significantly lower risk of developing metabolic syndrome than rural women and the low risks were observed in those who were less sitting and engaged in more vigorous physical activity (33). Moreover, randomized clinical trials have shown that interventions involving exercise were significantly associated with a reduced 
A

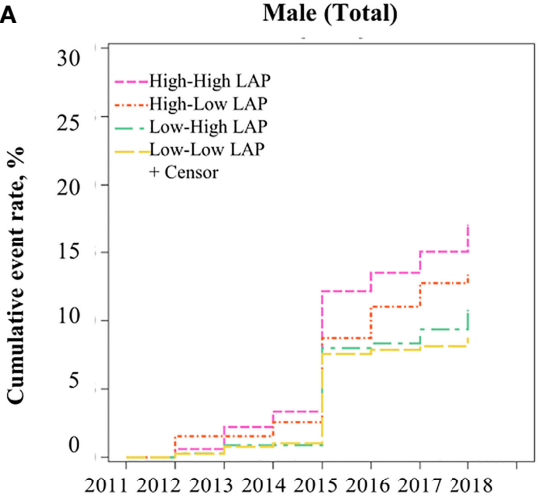

Number of cumulative events

$\begin{array}{lllllllll}\text { High-High } & 0 & 6 & 22 & 33 & 120 & 132 & 146 & 164\end{array}$ $\begin{array}{lllllllll}\text { High-Low } & 0 & 3 & 3 & 5 & 17 & 21 & 24 & 25\end{array}$ $\begin{array}{lllllllll}\text { Low-High } & 0 & 1 & 3 & 3 & 27 & 28 & 31 & 35\end{array}$ $\begin{array}{lllllllll}\text { Low-Low } & 0 & 2 & 6 & 8 & 59 & 61 & 63 & 67\end{array}$

C

Male (Rural)

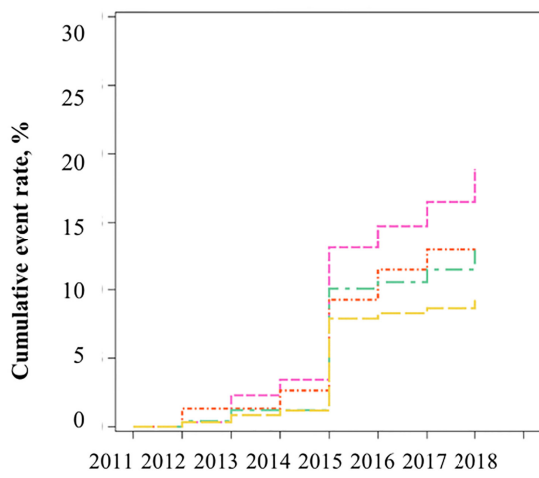
Number of cumulative events

$\begin{array}{lllllllll}\text { High-High } & 0 & 2 & 14 & 21 & 80 & 89 & 99 & 112\end{array}$ $\begin{array}{lllllllll}\text { High-Low } & 0 & 2 & 2 & 4 & 14 & 17 & 19 & 19\end{array}$ $\begin{array}{lllllllll}\text { Low-High } & 0 & 1 & 3 & 3 & 25 & 26 & 28 & 31\end{array}$ $\begin{array}{lllllllll}\text { Low-Low } & 0 & 2 & 5 & 7 & 47 & 49 & 51 & 54\end{array}$

E

Male (Urban)

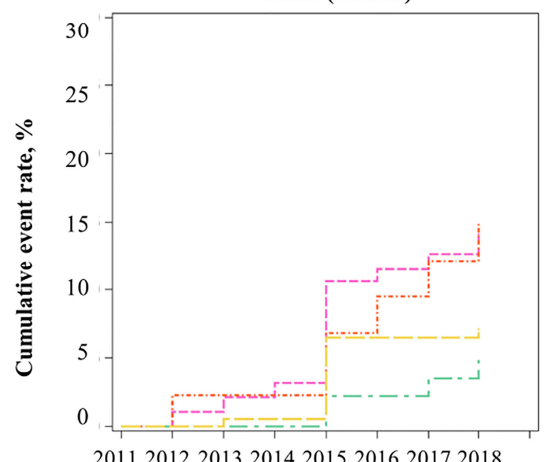

Number of cumulative events

$\begin{array}{llllclccc}\text { High-High } & 0 & 4 & 8 & 12 & 40 & 43 & 47 & 52 \\ \text { High-Low } & 0 & 1 & 1 & 1 & 3 & 4 & 5 & 6 \\ \text { Low-High } & 0 & 0 & 0 & 0 & 2 & 2 & 3 & 4 \\ \text { Low-Low } & 0 & 0 & 1 & 1 & 12 & 12 & 12 & 13\end{array}$

B

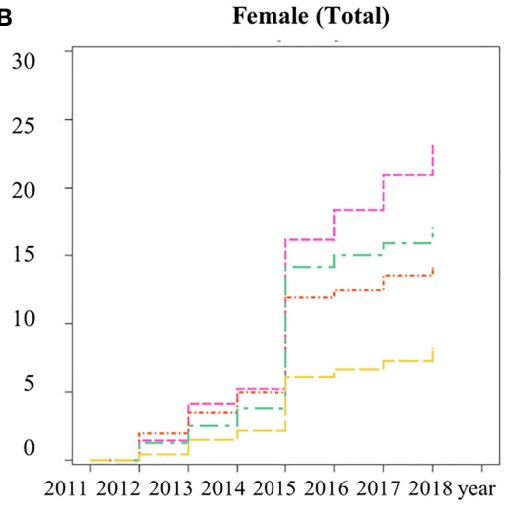

Number of cumulative events

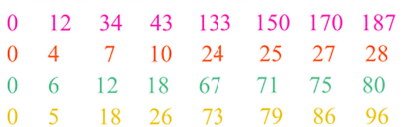

D

Female (Rural)

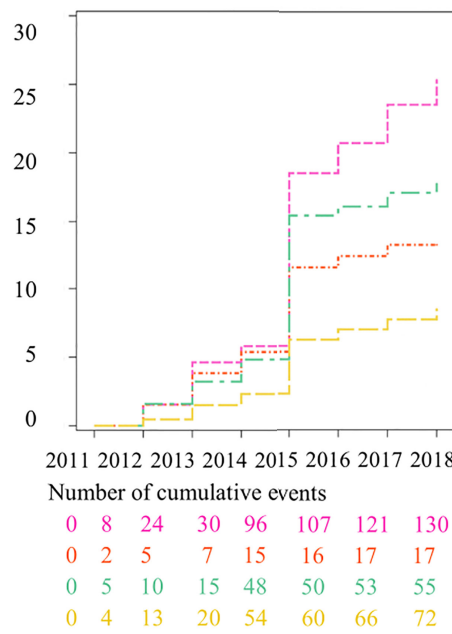

$\mathbf{F}$

Female (Urban)

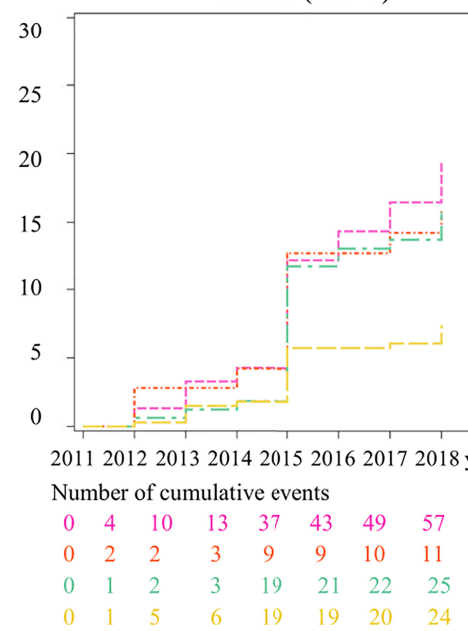

FIGURE 2 | Cumulative incidence of T2DM for LAP transitions from CHARLS 2011 to 2018. Note: LAP, lipid accumulation product; The cumulative event rate of T2DM was significantly different across four LAP transitions (high to high-, high to low-, low to high-, low to low-) in (A) Male, (B) Female, (C) Male in rural, (D) Female in rural, (E) Male in urban, and (F) Female in urban. 

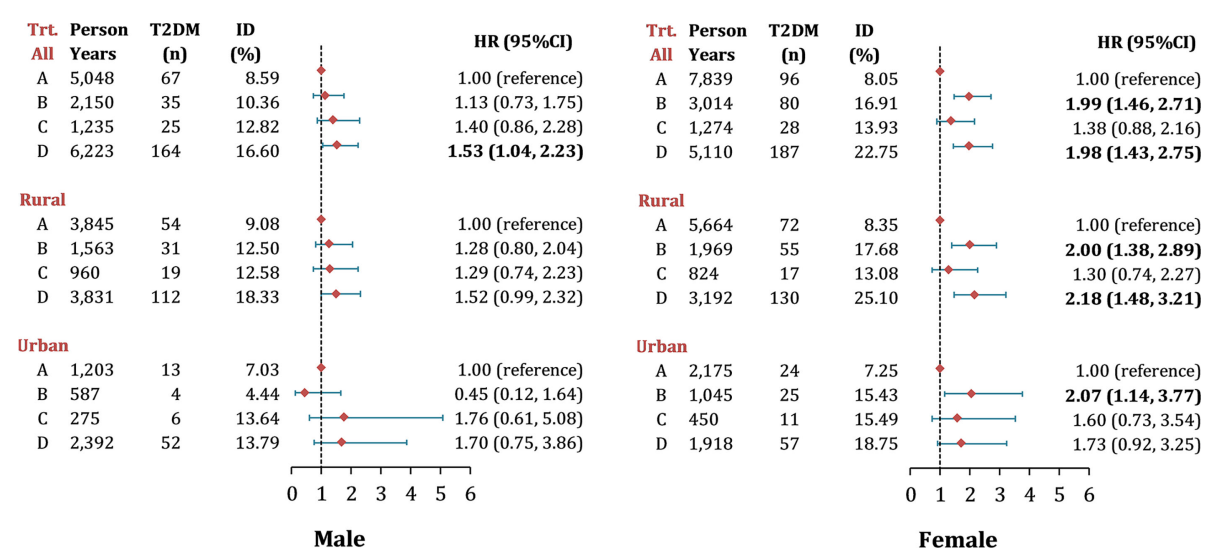

FIGURE 3 | Risk of new-onset T2DM by different LAP transitions in middle-aged and older Chinese. Note: Data were presented as n (\%) and hazard ratios (95\% $\mathrm{Cl}$ ), adjusted for age, education, region, hypertension, smoking, drinking, general obesity, TC, LDL-c level and HDL-c level; Hazard ratios for T2DM by LAP transitions were calculated using multivariable Cox frailty models with random effect, by which means clustering of participants was accounted for; $\mathrm{P}<0.05$ were highlighted in bold. ID, incidence density; LAP, lipid accumulation product; Trt., transition types during follow-up, the definition from group A to D were listed as following: Group A, maintain Low LAP during follow-up; Group B, Low LAP at baseline turned to High LAP at follow-up; Group C, High LAP at baseline turned to Low LAP at follow-up; Group D, maintain High LAP during follow-up.

risk of DM among people with prediabetes (34-36). Therefore, it is important that future research investigates the lifestyles of women in rural China, public health measures are also needed to mitigate the consequences of new cases of T2DM in this group.

Interestingly, for female participants, when assessing the impact of LAP transition on T2DM, the low-to-high LAP group had a similar risk for developing T2DM compared to the maintained-high LAP group, both groups were significantly higher than the maintained-low LAP group; comparatively, no significant difference was found between high-to-low LAP group and maintained-low LAP group. These findings may partly be explained by the "vicious circle" - the dyslipidemia-insulin resistance-hyperinsulinemia circle - in the development of T2DM (30). In this vicious circle, the consistently high level of TG, which leads to a maintained-high LAP, contributes to T2DM by competing with glucose to enter the cell, decreasing the activity of insulin receptors on fat cells, and preventing insulin from combining with receptors; moreover, the high LAP could also attribute to the decreased level of HDL, which can negatively influence the $\beta$ cell's function in the pancreas and reduce insulin sensitivity (31). Conversely, insulin resistance could result in the increasing of TG and decreasing of HDL. Given those, an increase in LAP from a low to a high level may be associated with the development of T2DM. Meanwhile, reducing and maintaining LAP at a lower level would be vital for the prevention of T2DM. Hence, further intervention studies could consider ways of shifting LAP levels as a way of preventing T2DM, especially for women.

This study is the first to analyze the impact of LAP and its transition on incident T2DM in middle to older aged population. Compared to previous studies, the sample of this study was relatively large, and representative based on participants from multiple regions in China. One of the limitations in the present study is that, due to the original design of the survey, blood sample data were only available in 2011 and 2015, which brought sudden increases in incident T2DM in 2015. Moreover, several confounding factors, such as family history and diet were not accounted for in our multivariable analyses due to the absence of relative information in the CHARLS dataset. Additionally, considering over half of the participants were excluded from this study, selection bias may be introduced.

This study demonstrated that high LAP was associated with the development of T2DM among Chinese women over 45 years old. The maintained high LAP and transition of LAP from low to high levels were confirmed as risk factors for T2DM. Future efforts aimed at preventing T2DM should be made to explore ways of decreasing LAP levels.

\section{DATA AVAILABILITY STATEMENT}

Publicly available datasets were analyzed in this study. This data can be found here: http://charls.pku.edu.cn/pages/data/111/en.html.

\section{ETHICS STATEMENT}

The studies involving human participants were reviewed and approved by Ethics Review Committee of Peking University. The patients/participants provided their written informed consent to participate in this study.

\section{AUTHOR CONTRIBUTIONS}

Conception or design: PS. Acquisition, analysis, or interpretation of data: GC, QY, and JY. Drafting the work or revising: JY, QY, YaS, YuS, LH, and YZ. Final approval of the manuscript: PS, YaS, YuS, LH, YZ, JY, and QY. All authors contributed to the article and approved the submitted version. 


\section{ACKNOWLEDGMENTS}

All authors thank the China Health and Retirement Longitudinal Study (CHARLS) for providing data. We are grateful for those who designed, conducted and participated in this study.

\section{REFERENCES}

1. Roglic G, Unwin N. Mortality Attributable to Diabetes: Estimates for the Year 2010. Diabetes Res Clin Pract (2010) 87:15-9. doi: 10.1016/j.diabres. 2009.10.006

2. I.D. Federation and IDF Diabetes Atlas. International Diabetes Federation. Brussels, Belgium: International Diabetes Federation (2019).

3. Chatterjee S, Khunti K, Davies MJ. Type 2 Diabetes. Lancet (2017) 389:223951. doi: 10.1016/S0140-6736(17)30058-2

4. Yuan H, Li X, Wan G, Sun L, Zhu X, Che F, et al. Type 2 Diabetes Epidemic in East Asia: A 35-Year Systematic Trend Analysis. Oncotarget (2018) 9:6718. doi: 10.18632/oncotarget.22961

5. He Y-H, Jiang G-X, Yang Y, Huang H-E, Li R, Li X-Y, et al. Obesity and Its Associations With Hypertension and Type 2 Diabetes Among Chinese Adults Age 40 Years and Over. Nutrition (2009) 25:1143-9. doi: 10.1016/j.nut.2009. 04.003

6. Ganguly S, Ray L, Kuruvila S, Nanda SK, Ravichandran K. Lipid Accumulation Product Index as Visceral Obesity Indicator in Psoriasis: A Case-Control Study. Indian J Dermatol (2018) 63:136. doi: 10.4103/ ijd.IJD_315_16

7. Bozorgmanesh, Mohammadreza, Farzad Hadaegh, Fereidoun Azizi. Predictive Performance of the Visceral Adiposity Index for a Visceral Adiposity-Related Risk: Type 2 Diabetes. Lipids Health Disease (2011) 10:1-9. doi: 10.1186/1476-511X-10-88

8. Du T, Yu X, Zhang J, Sun X. Lipid Accumulation Product and Visceral Adiposity Index are Effective Markers for Identifying the Metabolically Obese Normal-Weight Phenotype. Acta Diabetologica (2015) 52:855-63. doi: 10.1007/s00592-015-0715-2

9. Kahn HS. The Lipid Accumulation Product Is Better Than BMI for Identifying Diabetes: A Population-Based Comparison. Diabetes Care (2006) 29:151-3. doi: 10.2337/diacare.29.01.06.dc05-1805

10. Song J, Chen X, Jiang Y, Mi J, Zhang Y, Zhao Y, et al. Association and Interaction Analysis of Lipid Accumulation Product With Impaired Fasting Glucose Risk: A Cross-Sectional Survey. J Diabetes Res (2019) 2019:1-10. doi: $10.1155 / 2019 / 9014698$

11. Ray L, Ravichandran K, Nanda SK. Comparison of Lipid Accumulation Product Index With Body Mass Index and Waist Circumference as a Predictor of Metabolic Syndrome in Indian Population. Metab Syndrome related Disord (2018) 16:240-5. doi: 10.1089/met.2017.0119

12. Tian T, Pei H, Chen Z, Hailili G, Wang S, Sun Y, et al. Comparison of Lipid Accumulation Product and Body Mass Index as Indicators of Diabetes Diagnosis Among 215,651 Chinese Adults. PeerJ (2020) 8:e8483. doi: 10.7717/peerj.8483

13. Xia C, Li R, Zhang S, Gong L, Ren W, Wang Z, et al. Lipid Accumulation Product Is a Powerful Index for Recognizing Insulin Resistance in NonDiabetic Individuals. Eur J Clin Nutr (2012) 66:1035-8. doi: 10.1038/ ejcn.2012.83

14. Zhang LF, Pei Q, Yang GP, Zhao YC, Mu YF, Huang Q, et al. The Effect of IGF2BP2 Gene Polymorphisms on Pioglitazone Response in Chinese Type 2 Diabetes Patients. [Erratum Appears in Pharmacology. 2014;94(3-4):198]. Pharmacology (2014) 94:115-22. doi: 10.1159/000363414

15. Zhao Y, Strauss J, Yang G, Giles J, Hu P, Hu Y, et al. China Health and Retirement Longitudinal Study-2011-2012 National Baseline Users' Guide. Beijing: National School of Development, Peking University (2013) p. $1-56$.

16. W.H. Organization. Physical Status: The Use of and Interpretation of Anthropometry, Report of a WHO Expert Committee. Geneva, Switzerland: World Health Organization (1995).

17. Deaton A. The Analysis of Household Surveys: A Microeconometric Approach to Development Policy. Washington: The World Bank (1997).

\section{SUPPLEMENTARY MATERIAL}

The Supplementary Material for this article can be found online at: https://www.frontiersin.org/articles/10.3389/fendo.2021.770200/ full\#supplementary-material

18. Song P, Wang H, Xia W, Chang X, Wang M, An L. Prevalence and Correlates of Hyperuricemia in the Middle-Aged and Older Adults in China. Sci Rep (2018) 8:4314. doi: 10.1038/s41598-018-22570-9

19. James PA, Oparil S, Carter BL, Cushman WC, Dennison-Himmelfarb C, Handler J, et al. Evidence-Based Guideline for the Management of High Blood Pressure in Adults: Report From the Panel Members Appointed to the Eighth Joint National Committee (JNC 8). JAMA (2014) 311:507-20. doi: 10.1001/ jama.2013.284427

20. Chen C, Lu FC. The Guidelines for Prevention and Control of Overweight and Obesity in Chinese Adults. Biomed Environ Sci BES (2004) 17 Suppl:1-36.

21. American Diabetes Association. Diagnosis and Classification of Diabetes Mellitus. Diabetes Care (2014) 37:S81-S90. doi: 10.2337/dc14-er03

22. Pan X-R, Yang W-Y, Li G-W, Liu J. Prevalence of Diabetes and Its Risk Factors in China, 1994. Diabetes Care (1997) 20:1664-9. doi: 10.2337/ diacare.20.11.1664

23. Gu D, Reynolds K, Duan X, Xin X, Chen J, Wu X, et al. Prevalence of Diabetes and Impaired Fasting Glucose in the Chinese Adult Population: International Collaborative Study of Cardiovascular Disease in Asia (InterASIA). Diabetologia (2003) 46:1190-8. doi: 10.1007/s00125-003-1167-8

24. Jia W, Pang C, Chen L, Bao Y, Lu J, Lu H, et al. Epidemiological Characteristics of Diabetes Mellitus and Impaired Glucose Regulation in a Chinese Adult Population: The Shanghai Diabetes Studies, a Cross-Sectional 3-Year Follow-Up Study in Shanghai Urban Communities. Diabetologia (2007) 50:286-92. doi: 10.1007/s00125-006-0503-1

25. Yang W, Lu J, Weng J, Jia W, Ji L, Xiao J, et al. Prevalence of Diabetes Among Men and Women in China. New Engl J Med (2010) 362:1090-101. doi: 10.1056/NEJMoa0908292

26. Bozorgmanesh M, Hadaegh F, Azizi F. Diabetes Prediction, Lipid Accumulation Product, and Adiposity Measures; 6-Year Follow-Up: Tehran Lipid and Glucose Study. Lipids Health Dis (2010) 9:45. doi: 10.1186/1476511X-9-45

27. Nusrianto R, Ayundini G, Kristanti M, Astrella C, Amalina N, Riyadina W, et al. Visceral Adiposity Index and Lipid Accumulation Product as a Predictor of Type 2 Diabetes Mellitus: The Bogor Cohort Study of Non-Communicable Diseases Risk Factors. Diabetes Res Clin Pract (2019) 155:107798. doi: 10.1016/j.diabres.2019.107798

28. Xu M, Huang M, Qiang D, Gu J, Li Y, Pan Y, et al. Hypertriglyceridemic Waist Phenotype and Lipid Accumulation Product: Two Comprehensive Obese Indicators of Waist Circumference and Triglyceride to Predict Type 2 Diabetes Mellitus in Chinese Population. J Diabetes Research (2020) 2020:1-12. doi: 10.1155/2020/9157430

29. Wakabayashi I, Daimon T. A Strong Association Between Lipid Accumulation Product and Diabetes Mellitus in Japanese Women and Men. J Atheroscl Thromb (2013) 21(3):20628. doi: 10.5551/jat.20628

30. Liu X, Li Y, Li L, Zhang L, Ren Y, Zhou H, et al. Prevalence, Awareness, Treatment, Control of Type 2 Diabetes Mellitus and Risk Factors in Chinese Rural Population: The RuralDiab Study. Sci Rep (2016) 6:1-9. doi: 10.1038/ srep31426

31. Blaak E. Sex Differences in the Control of Glucose Homeostasis. Curr Opin Clin Nutr Metab Care (2008) 11:500-4. doi: 10.1097/MCO.0b013e32830467d3

32. Fuente-Martín E, Argente-Arizón P, Ros P, Argente J, Chowen JA. Sex Differences in Adipose Tissue: It Is Not Only a Question of Quantity and Distribution. Adipocyte (2013) 2:128-34. doi: 10.4161/adip.24075

33. Xiao J, Shen C, Chu MJ, Gao YX, Xu GF, Huang JP, et al. Physical Activity and Sedentary Behavior Associated With Components of Metabolic Syndrome Among People in Rural China. PloS One (2016) 11:e0147062. doi: 10.1371/ journal.pone. 0147062

34. Tuomilehto J, Lindström J, Eriksson JG, Valle TT, Hämäläinen H, IlanneParikka P, et al. Prevention of Type 2 Diabetes Mellitus by Changes in 
Lifestyle Among Subjects With Impaired Glucose Tolerance. New Engl J Med (2001) 344:1343-50. doi: 10.1056/NEJM200105033441801

35. Group DPPR. Reduction in the Incidence of Type 2 Diabetes With Lifestyle Intervention or Metformin. New Engl J Med (2002) 346:393-403. doi: 10.1056/ NEJMoa012512

36. Li G, Zhang P, Wang J, Gregg EW, Yang W, Gong Q, et al. The Long-Term Effect of Lifestyle Interventions to Prevent Diabetes in the China Da Qing Diabetes Prevention Study: A 20-Year Follow-Up Study. Lancet (2008) 371:1783-9. doi: 10.1016/S0140-6736(08)60766-7

Conflict of Interest: The authors declare that the research was conducted in the absence of any commercial or financial relationships that could be construed as a potential conflict of interest.
Publisher's Note: All claims expressed in this article are solely those of the authors and do not necessarily represent those of their affiliated organizations, or those of the publisher, the editors and the reviewers. Any product that may be evaluated in this article, or claim that may be made by its manufacturer, is not guaranteed or endorsed by the publisher.

Copyright () $2021 \mathrm{Yu}, \mathrm{Yi}$, Hou, Chen, Shen, Song, Zhu and Song. This is an open-access article distributed under the terms of the Creative Commons Attribution License (CC BY). The use, distribution or reproduction in other forums is permitted, provided the original author(s) and the copyright owner(s) are credited and that the original publication in this journal is cited, in accordance with accepted academic practice. No use, distribution or reproduction is permitted which does not comply with these terms. 